Asian Spine Journal

Vol. 3, No. 2, pp 96 100, 2009

\title{
The Clinical Effect of Gait Load Test in Two Level Lumbar Spinal Stenosis
}

\author{
Youn-Soo Kim, Sung-Jin Park, In-Soo Oh, Jae-Young Kwan \\ Department of Orthopedics, The Catholic University of Korea College of Medicine, Seoul, Korea.
}

Study Design: This study is a prospective, clinical study assessing the efficacy of selective decompression of the level responsible in a two-level stenosis in accordance with the neurological findings defined by the gait load test with a treadmill.

Purpose: To clarify the clinical features of multilevel lumbar spinal stenosis (LSS) regarding the neurological level responsi ble for the symptoms, neurogenic claudication, and outcomes of selective decompression.

Overview of Literature: Most spine surgeons have reported that multilevel compression of the cauda equina induces a more severe impairment of the nerve function than a single-level compression. However, the clinical effects of multilevel LSS on the cauda equine and nerve roots are unknown.

Methods: A total of 21 patients with lumbar spinal canal stenosis due to spondylosis and degenerative spondylolisthesis were selected. The level responsible for the symptoms in the two-level stenosis was determined from the neurological findings on the gait load test and functional diagnosis based on a selective nerve root block. All patients underwent a prospective, selective decompression at the level neurologically responsible only. The average follow-up period was 2.6 years (range, 1 to 6 years). The postsurgical outcome was defined using the Visual Analogue Scale (VAS) at the post-gait load test, 2 weeks after surgery, 3 months after surgery and at the last follow up.

Results: Before surgery, the mean threshold distance and mean walking tolerance was $34.3 \mathrm{~m}$ and $113 \mathrm{~m}$, respectively. All patients had neurogenic claudication and 19 of the patients had cauda equina syndrome, including hypesthesia in 11 cases, muscle weakness in 5 cases and radicular pain in 7 cases. Selective nerve blocks to determine the level responsible for the lumbosacral symptoms in 2 cases revealed a mean VAS score of 7.1, 2.61, 3.04, and 3.47 at the post-gait load test, 2 weeks after surgery, 3 months after surgery and at the last follow up, respectively. All subjects underwent surgery. After the operation, neurogenic claudication with or without cauda equna syndrome subsided in all patients

Conclusions: The gait load test allows an objective and quantitative evaluation of the gait characteristics of patients with lumbar canal stenosis and is useful for determining the appropriate level for surgical treatment.

Key Words: Gait load test, Neurogenic claudication, Lumbar canal stenosis

\section{Introduction}

Lumbar spinal stenosis (LSS) is defined as any type of narrowing of the spinal canal, nerve root canal, or interver- tebral foramen ${ }^{1}$. In the pathogenesis of LSS, the degenerative process of the lumbar spine is the main component that eventually compresses the neural tissue in the spinal and/or nerve root canal ${ }^{2}$. The typical symptoms of LSS include neurogenic claudication (NC), back and leg pain and mixed

Received Oct 29, 2009; 1st revised Nov 18, 2009; accepted Nov 18, 2009

Corresponding author: Sung-Jin Park, MD

St. Paul's Hospital, Jeonnong 2-dong, Dongdaemun-gu, Seoul 130-709, Korea

Tel: +82-2-958-2158, Fax: +82-2-966-5158, E-mail: sungjinp@ catholic.ac.kr 
symptoms ${ }^{3}$. NC limits walking in patients with LSS because after walking a short distance, the patients experience weakness, tiredness, or heaviness of the legs that increases, gradually causing them to stop. A suspected pathophysiological mechanism underlying $\mathrm{NC}$ is intermittent hypoxia of the cauda equina fibers that can lead to ischemic nerve conduction failure and transient clinical and electrophysiological changes after exercise $e^{4}$. The exercise treadmill test is used to confirm the complaints experienced by patients while walking.

The diagnosis of LSS is based on the results of a clinical examination and radiological documentation of the narrowing of the lumbar spinal canal ${ }^{5,6}$. The clinical differentiation between lumbosacral polyradicular disease (typical of LSS) and generalized peripheral neuropathies of the distal-axonal type (typical of diabetic patients) can be difficult ${ }^{7}$. This study examined the clinical features of multilevel LSS with regard to the types of $\mathrm{NC}$ and the levels responsible for the neurologic symptoms using the neurologic findings defined by the gait load test with a treadmill. On addition, the effi- cacy of selective one level decompression of only the level responsible for the symptoms in multilevel LSS was assessed.

\section{Materials and Methods}

Twenty one subjects were selected from patients with lumbar spinal canal stenosis confirmed by a plain X-ray,

Table 1. Selected patients

\begin{tabular}{lc}
\hline \hline Study and level of spinal stenosis & No. \\
\hline Imaging technique & 16 \\
Myelogram \& CT & 4 \\
MRI & 1 \\
Myelogram \& CT, MRI & 17 \\
\hline Level of lumbar spinal stenosis (LSS) & 4 \\
LSS 3-4-5
\end{tabular}
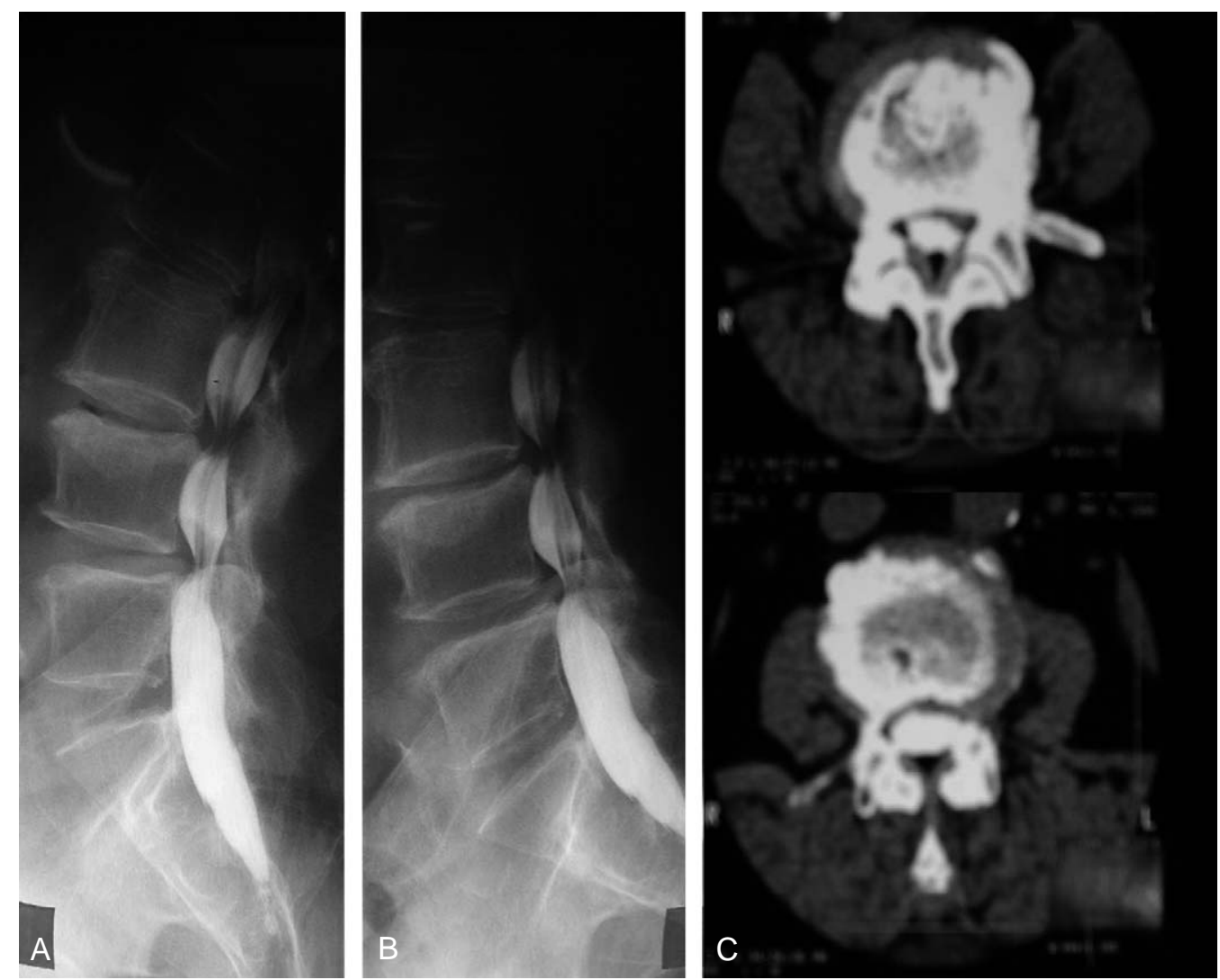

Fig. 1. (A) Preoperative myelogram. (B) Preoperative CT finding (L3-4). (C) Preoperative CT findings (L4-5). A 65 year old female patient underwent a gait load test. After checking the radicular pain to the left calf and the left big toe dorsiflexion weakness, a total laminectomy at L4-5 was performed. The Visual Analogue Scale score in the post-gait load test was 7 and 3 at the last follow up. 
MRI, myelo-CT (MRI in 4 cases, myelo-CT in 16 cases, and MRI combined with myelo-CT in 1 case). All subjects had intermittent claudication while visiting the department. The selection criteria were a history suggesting nerve root type neuropathy with unilateral leg pain or numbness, or a history suggestive of cauda equina type neuropathy with the symptoms of cauda equina and bilateral leg pain or numbness. The patients were excluded if they had symptoms that were severe at rest, other diseases that could affect walking, or no symptoms suggestive of cauda equina syndrome despite the presence of bilateral leg symptoms.

All 21 subjects ( 7 men and 14 women with a mean age of 59.2 years) underwent a gait load test. According to the international classification, neuropathy in the nerve root type group was rated as degenerative in 17 cases, and spondylolisthesis in 4 cases (Table 1).

An ambulatory treadmill test with a speed of $2 \mathrm{~km} /$ hour was carried out for the gait load test. The investigator checked the distance from the start of the treadmill test to the onset of spinal claudication symptoms (threshold distance), and each patient decided when to end the test (walking tolerance). The result was considered to be good if the threshold distance was above $250 \mathrm{~m}$ after surgery.

The investigator did not encourage or influence the patient in any way. The investigator then checked which symptoms had worsened and performed a quick neurological examination to determine if the sensory and motor deficits had worsened. A gait load test can make latent neurogenic abnormalities and symptoms apparent. A more precise neurological evaluation of patients with $\mathrm{NC}$ is possible using the gait load test. All patients underwent selective decompression (total or hemi laminectomy and foraminotomy) at the neurologically responsible level only (Fig. 1). The postsurgical outcome was defined using a Visual Analogue Scale (VAS) at the post-gait load test, 2 weeks after

Table 2. Neurologic symptom after gait load test

\begin{tabular}{lc}
\hline \hline Neurologic symptom & No. \\
\hline Hypesthesia on L5 dermatome & 11 \\
Big toe dorsiflexion & 5 \\
Radicular pain & 7 \\
\hline
\end{tabular}

surgery, 3 months after surgery, and at the last follow up. The authors checked the neurological symptoms and NC of the selected level in current studies before and after surgery. The VAS, which is a subjective measure, was selected because the postoperative results could not be assessed objectively.

\section{Results}

Before surgery, the mean threshold distance and mean walking tolerance was $34.3 \mathrm{~m}$ (5 to $151 \mathrm{~m})$ and $113 \mathrm{~m} \mathrm{(24}$ to $253 \mathrm{~m}$ ), respectively. Before surgery, all patients had NC and nineteen had cauda equna compression symptoms including hypesthesia in 11 cases, muscle weakness in 5 in cases and radicular pain in 7 cases (Table 2).

The selective nerve blocks to determine the level responsible for the lumbosacral symptoms was performed in 2 cases. After surgery, $\mathrm{NC}$ and cauda equina syndrome were improved in all patients. The patients' threshold distance was $>250 \mathrm{~m}$, which means good results after one level decompression in multilevel LSS.

The mean VAS score at the post-gait load test, immediately after surgery, 2 weeks, 3 months, and at the last follow up was 7.1, 2.61, 3.04, and 3.47, respectively (Table 3). Three patients reported a VAS score $>6$ at the last follow up. One patient had a compression fracture due to osteoporosis and other two complained of low back pain due to progressing degenerative spondylosis. Good results were obtained from the gait load test with the treadmill, which suggests that it may be good method for determining the level in multilevel LSS.

\section{Discussion}

Although lumbar spinal stenosis is one of the most common reasons for spinal intervention ${ }^{8}$, its clinical and radiological signs leading to an indication for surgery have not been clearly defined ${ }^{9,10}$. Decompressive surgical procedures for multilevel lumbar spinal stenosis include laminectomy, hemilaminotomy, fenestration, foraminotomy and the

Table 3. Visual Analogue Scale score

\begin{tabular}{cccc}
\hline \hline Preoperation & Postoperation 2 wk & Postoperation 3 mon & Last follow up \\
\hline 7.19 & 2.61 & 3.04 & 3.47 \\
\hline
\end{tabular}


implantation of interspinous distraction devices. The complication rates for decompression surgery (intra and postoperation) range from $14 \%$ to $35 \%$ or higher ${ }^{11,12}$. Fusion surgery, which is a more invasive procedure than decompression surgery, is associated with higher complication rates. Typical complications of both decompression and fusion surgery include dura vessel lacerations, epidural hematomas, inadequate decompression with significant residual stenosis, instability and reossification. All of these complications resulted in renewed nerve compression ${ }^{13}$. The authors examined the results of the gait load test with a treadmill to avoid instability and minimize the number of complications after surgery.

The difficulty in making a diagnosis lies in the frequent absence of clinical symptoms at rest, while enormous pain and limited function have been described under physical strain. The additional diagnostics based on the X-ray, CT, MRI, Myelography, and Myelo-CT are not sufficiently predictive of the severity of a stenosis9.

$\mathrm{NC}$ is described as the classic clinical presentation of LSS, a degenerative condition of the lumbar spine normally affecting adults over the age of 50. The symptoms of NC are described as pain, paraesthesia or cramping of one or both legs, which are induced while walking and relieved when sitting ${ }^{4}$. The effect of posture on the symptoms is the primary distinguishing feature of NC. The symptoms are typically exacerbated when the spine is extended (in an upright stance when standing or walking) and eased when the spine is flexed (stooping forwards or sitting) ${ }^{14}$. The clinical symptoms are believed to result from stenotic changes (narrowing) that are exacerbated by posture-related compression, causing neural and microvascular compromise of the cauda equina and lumbosacral nerve roots ${ }^{4}$. It was reported that these findings have high sensitivity in identifying patients with radiological stenosis but the specificity is variable.

In order to minimize the surgical procedure, only one level of a symptomatic multilevel LSS was decompressed after the gate load test was performed using a treadmill. Decompression of the selected level in multilevel LSS produced good results.

It is possible to treat patients with multilevel LSS to decompress one selected level, because $\mathrm{NC}$ is present in the most severe compressed vertebral canal and/ or vertebral foramen.

A recent review reported that no firm conclusions on the diagnostic performance of clinical or radiological tests can be made ${ }^{15}$. On the other hand, the clinical syndrome of NC, provides a recognizable and meaningful subgroup of chronic low back pain patients, who can be identified by the presence of posture-related clinical symptoms.

The Gait load test helps objectify pre- and postsurgical clinical complaints and verifies a lumbar spinal stenosis by creating a situation of dynamic strain. An assessment of the orthopedic and neurological status by an independent examiner at the end of the test delivers additional diagnostic certainty.

It is believed that expensive spinal imaging may be unnecessary except where surgery is planned.

\section{Conclusions}

The gait load test allows an objective and quantitative evaluation of the gait characteristics of patients with lumbar canal stenosis and is useful for determining the levels for surgical treatment in these patients. In addition, it can be another less invasive treatment with minimal post surgical complications.

\section{REFERENCES}

1. Arnoldi CC, Brodsky AE, Cauchoix J, et al: Lumbar spinal stenosis and nerve root entrapment syndromes: definition and classification. Clin Orthop Relat Res 1976; (115): 4-5.

2. Airaksinen O, Herno A, Turunen V, Saari T, Suomlainen O: Surgical outcome of 438 patients treated surgically for lumbar spinal stenosis. Spine (Phila Pa 1976) 1997; 22: 2278-2282.

3. Johnsson KE, Willner S, Pettersson H: Analysis of operated cases with lumbar renal stenosis. Acta Orthop Scand 1981; 52: 427-433.

4. Porter RW: Spinal stenosis and neurogenic claudication. Spine (Phila Pa 1976) 1996; 21: 2046-2052.

5. Getty CJ: Lumbar spinal stenosis: the clinical spectrum and the results of operation. J Bone Joint Surg Br 1980; 62: 481-485.

6. Uden A, Johnsson KE, Jonsson K, Pettersson H: Myelography in the elderly and the diagnosis of spinal stenosis. Spine (Phila Pa 1976) 1985; 10: 171-174.

7. Adamova B, Vohanka S, Dusek L: Differential diagnostics in patients with mild lumbar spinal stenosis: the contri- 
butions and limits of various tests. Eur Spine J 2003; 12: 190-196.

8. Katz JN: Lumbar spinal fusion: surgical rates, costs, and complications. Spine (Phila Pa 1976) 1995; 20: 78S-83S.

9. Amundsen T, Weber $\mathbf{H}$, Lilleas $\mathbf{F}$, et al: Lumbar spinal stenosis: clinical and radiologic features. Spine (Phila $\mathrm{Pa}$ 1976) 1995; 20: 1178-1186.

10. Yukawa Y, Lenke LG, Tenhula J, et al: A comprehensive study of patients with surgically treated lumbar spinal stenosis with neurogenic claudication. J Bone Joint Surg Am 2002; 84: 1954-1959.

11. Costa F, Sassi M, Cardia A, et al: Degenerative lumbar spinal stenosis: analysis of results in a series of 374 patients treated with unilateral laminotomy for bilateral microdecompression. J Neurosurg Spine 2007; 7: 579-586.
12. Kim DH, Albert TJ: Interspinous process spacers. J Am Acad Orthop Surg 2007; 15: 200-207.

13. Benz RJ, Ibrahim ZG, Afshar P, Garfin SR: Predicting complications in elderly patients undergoing lumbar decompression. Clin Orthop Relat Res 2001; (384): 116121.

14. Takahashi K, Kagechika K, Takino T, et al: Changes in epidural pressure during walking in patients with lumbar spinal stenosis. Spine (Phila Pa 1976) 1995; 20: 27462749.

15. de Graaf I, Prak A, Bierma-Zeinstra S, et al: Diagnosis of lumbar spinal stenosis: a systematic review of the accuracy of diagnostic tests. Spine (Phila Pa 1976) 2006; 31: 1168-1176. 